2. - Note on cubic equations in three variables with an infinity of integer solutions, Annali di Matematica Pura ed Applicata (4) vol. 29 (1949) pp. 301-305; or Colloques Internationaux du Centre National de la Recherche Scientifique, vol. 24, Paris, 1950, pp. 77-79.

3. B. Segre, Sur les points entiers des surfaces cubiques, Colloques Internationaux du Centre National de la Recherche Scientifique, vol. 24, Paris, 1950, pp. 81-82.

St. John's College, Cambridge University

\title{
ON THE SET OF VALUES OF A NONATOMIC, FINITELY ADDITIVE, FINITE MEASURE
}

\author{
R. J. NUNKE AND L. J. SAVAGE
}

A countably additive, nonatomic, finite measure takes on every value from zero to its maximum, inclusive, where, as throughout this note, it is to be understood that measures are non-negative. ${ }^{1}$ The purpose of this note is to exhibit a counter-example, expressed as a theorem, which shows that finitely additive measures are as queer in this respect as in many others. ${ }^{2}$

THEOREM. If a Boolean algebra $X$ with identity $X$ carries any finitely additive, nonatomic measure at all, it carries one such measure, say $m$, such that $m(X)=4$, but none of the values of $m$ lie in the interval $(1,3)$.

Proof. Let $p$ be a nonatomic, finitely additive measure. Without loss of generality it may be assumed that $p(X)=1$.

By Zorn's Lemma there exists a nonvacuous subset $\Upsilon$ of $X$ maximal with respect to the properties:

1. If $p(A)=0, A \in \Upsilon$.

2. If $A, B \in \Upsilon, A \cup B \in \Upsilon$.

3. If $A$ is in $\Upsilon$ and $B$ is in $X, A \cap B \in \Upsilon$.

That is, there is a maximal ideal containing the ideal of elements of $p$-measure 0 . Denote the complement of $\Upsilon$ by $Z$. In virtue of its maximality with respect to properties $1-3, \Upsilon$ has also the following properties:

Received by the editors February 26, 1951.

${ }^{1}$ See for example Lemma 2 of P. R. Halmos. On the set of values of a finite measure, Bull. Amer. Math. Soc. vol. 53 (1947) pp. 138-144.

${ }^{2}$ Attention is called to the papers of A. Sobczyk and P. C. Hammer, Duke Math. J. vol. 11 (1944) pp. 839-846 and pp. 847-851 respectively, which are closely related to and more extensive than the present note but do not happen to cover the same point. 
4. If $A \in \Upsilon, X-A \in \mathcal{Z}$.

5. If $A \cap B=0, A \in \Upsilon$ or $B \in \Upsilon$.

Consider now the set function $m$ such that

$$
\begin{array}{ll}
m(A)=p(A) & \text { for } A \in \Upsilon, \\
m(A)=3+p(A) & \text { for } A \in \mathcal{Z} .
\end{array}
$$

Properties 1-5 imply easily that $m$ satisfies the conclusion of the theorem.

It may be of some interest to remark before closing that since the analogous theorem for countably additive measures is false, there cannot exist in a $\sigma$-algebra a maximal $\sigma$-ideal containing all elements on which a countably additive nonatomic measure vanishes, where by a $\sigma$-ideal, we mean an ideal closed under countable union.

University of Chicago 